\title{
Septicemia intrahospitalaria por Acinetobacter calcoaceticus variedad anitratus en una unidad de cuidados intensivos neonatales
}

\author{
Guillermo Vivanco G. ${ }^{\mathbf{1}}$; Adela Figueroa B.' \\ Nosocomial neonatal sepsis by Acinetobacter calcoaceticus \\ biovar anitratus
}

Twenty one cases of sepsis by Acinetobacter calcosceticus biovar anitratus occurred, ten in 1989 and eleven in 1990 (januaryapril). In this tast year, there were nine cases with characteristics of an epidemic outbreak. It was noted that sepsis was predominant in males, babies of less than $2500 \mathrm{~g}$ and less than 37 weeks of gestation. They were hospitalized in our Intensive Care Unit for different problems, but almost all had mechanical ventilation. parenteral nutrition and received antibiotics previously, being these risk factors the most important. Clinical and laboratory features were not different from sepsis produced by other microorganisms, It is important to remark the increased frecuency of Acinetobacter caicoaceticus biovar anitratus as a nosocomial agent, but also his greater aggressitivity and antibiotic resistance, all factors that produced an elevated mortality, eight of eleven cases. Finally. due to the adoption of several measures the epidemic outbreak was stopped.

[Key words: Acinetobacter infections, septıcemia, newborn.]

1. Unidad de Neonatología, Servicio de Pediatría, Hospital Félix Bulnes Cer da.

Durante los meses de marzo a abril de 1990 un brote de septicemias intrahospitalarjas, provocadas por Acinetobacter calcoaceticus, varie- 
dad anitratus (ACA), causó varias muertes en nuestra unidad de cuidados intensivos neonatales. Si bien ACA es conocido desde hace tiempo como un agente etiológico de infecciones nosocomíales en adultos ${ }^{1}$, niños y recién nacidos ${ }^{2,3}$, en su carácter de agente oportunista no produce habitualmente problemas de la gravedad que tuvieron los episodios que nos motivaron a realizar esta descripción. El ACA asume en la tinción de Gram la forma de cocobacilo o diplo. coco Gram negativo y, en líneas generales, se puede decir que se identifica en el laboratorio por sus características negativas, ya que es no fermentador, no reduce nitrato y es inmóvil4.

El propósito de este estudjo fue describir las características epidemiológicas y clínicas de los casos ocurridos, como también revisar y comparar los sucedido con este agente en el año precedente (1989), pues pareció que en 1990 adquiria características diferentes, de mayor gravedad y letalidad.

\section{Material y Método}

Se revisaron las fichas clínicas de todos los recién nacidos que estuyieron hospitalizados en la unidad de cuidados intensivos neoratales del hospital Félix Bulnes, entre enero de 1989 y abril de 1990 , que presentarón en algún momento de su evolución uno o más hemocultivos positivos a ACA, con o sin cultivos positivos de líquido cefalorraquídeo, en quienes se sospechó, concomitantemente y sobre bases clínicas, que sufráan septicemia ${ }^{5}$. Todos habian sido hospitalizados desde la maternidad del nisno hospital, directamente en la unidad, por diversas causas, tomándoseles muestras para hemocultivos desde venas o atterjas periféricas del pliegue del codo, con técnicas de enfermería estándar6. La sangre extraj́da para los hemocultivos fue sembrada directamente en medio corriente de infusión cerebro-corazón, desde donde se tomaron muestras para sembrar en agar sangre y Mackonkey, tinción de Gram -que permitió identificar al ACA como cocobacilo o diplococo Gram negativo-, realizar siembras en medios selectivos y las pruebas específicas para su determinación. A todas las cepas identificadas como ACA se les había practicado antíbiograma por difusión en agar por el método de Kirby Bauer ${ }^{7}$ e investigado su sensibilidad a diferentes antibióticos, de acuerdo a los sensidiscos disponibles: ampicitina, gentamicina, cotrimoxazol, amikacina, cefradina, ceftrinxona, ampicjlina-sulbactam, aztreonam, cefotaxima, ciprofloxacín, cefoperazona, ceftazidima y ceftizoxima. Se usaron tabletas 0 sensidiscos Arlab ${ }^{(E)}$ y S.D.A. ${ }^{(B)}$. Se consideraron positivos y se incorporaron al estudio sólo casos en que se aisló el ACA como microorganismo único en uno o más hemocultivos, elinninando a los que tenian cultivos polimicrobianos. En todos los recién nacidos admitidos al estudio se hizo un completo registro de los datos de la fícha clínica, incluyendo antecedentes mater. nos, obstétricos y del recién nacido, sus exámenes de laboratorio, tratamiento y evolución. La interpretación del hemograma se realizó de acuerdo a los criterios de Manroe y cols ${ }^{3}$, e incluyó recuento de glóbulos rojos, glóbulos blancos, fórmula diferencial, índice de formas inmaduras versus neutrófilos totales y recuento de plaquetas. Durante el período de mayor frecuencia de ajslamientos de ACA se tomaron muestras para cultivos del personal médico, paramédico y del medio ambiente, incluyendo laringoscopios, incubadoras, oxímetros, equipos manuales de reanimación, ventiladores mecánicos, monitores $y$, en general, de todos $10 \mathrm{~s}$ instrumentos que hubiesen estado en contacto con los recién nacidos infectados y pudiesen haber contribuido a la điseminación de la infección con el propósito de investigna su origen y controlarla.

\section{Resultados}

En 1989 hubo diez casos que cumplían los requisitos del estudio a lo largo de todo el año, pero entre los meses de enero y abril del año siguiente (1990) se presentaron once, nueve de los cuales ocurrieron en marzo $(n=5)$ y abril $(n=4)$. Las características más relevantes de los 21 casos son las siguientes: 14 eran hombres, 15 pesaban menos de $2500 \mathrm{~g}$ al nacer $(6$ menos de 1500 g) y 13 tenían menos de 37 semanas de edad gestacional ( 7 menos de 32 semanas), 12 nacjeron por parto vaginal y en 5 de los 21 la calificación de Apgar era menor o igual a 5 a los 5 minutos. En el afto 1989 transcurrieron en promedio 12 días desde el momento del ingreso a la unidad hasta la aparición del hemocultivo positivo a ACA, mediana 10 días y letalidad $20 \%$ ( 2 casos). En contraste, en el año 1990 el tiempo de latencia promedio fue de sólo 6,3 días, la mitad de los casos aparecieron en los primeros 5 días de hospitalización y la letalidad subió a 8 de 11 niños infectados (72,7\% ).

Todos los casos se asociaron ya sea con ventilación mecánica, alimentación parenteral o tratamiento antibiótico previo y $\operatorname{los} 3$ factores estuvieron presentes en 18 de los 21 casos. Cinco se asociaron con cateterización umbilical y 8 con procedimientos quirúrgicos.

Las características clínicas de los pacientes en sepsis intrahospitalarias por ACA se describen en la tabla y fueron semejantes en ambos aftos, destacando los síntomas clásicos de color terroso en 18 nifíos, alteración de la termorregulación en 16, mala tolerancia oral y asociación con neumonía en 14. Sólo en el brote del año 1990 tuvimos 4 casos con choque séptico, todos fatales. En el hemograma cabe destacar que hu- 
bo desviación a izquierda con índjce $\mathrm{I} / \mathrm{T}$ mayor o igual a 0,2 en 14 casos y más de 15000 baciliformes por $\mathrm{mm}^{3}$ en 15 de ellos. La plaquetopenia, menor de 150000 apareció en 12 de los 21 casos analizados. Diez nifíos tuvieron leucocitosis $\geqslant 25000 \mathrm{x} \mathrm{mm}^{3}, 4$ tuvieron $\leqslant 6000$ leu. cocitos $x \mathrm{~mm}^{3}$.

El comportamiento del germen frente a los antibióticos fue distinto en el año 1989 y en 1990. En el primer caso el germen fue sensible a la amikacina, gentamicina, cefradina y cloran. fenicol; en 1990 el gemnen fue sensible sólo a ampicilina-sulbactam, llegando a ser, en una ocasión, sólo medianamente sensible a éste y resistente a todos los demás.

Ante la evidencia de un brote epidémico se tomaron varias medidas, entre ellas el cierre de la unidad de cuidados intensivos neonatales por 15 dias, aislamiento estricto de los casos, uso de medidas de circulación y técnicas de enfermería destinadas a evitar la propagación?. Se tomaron muestras de cultivos de manos a todo el personal médico y paramédico; al microam. biente del recién nacido incluyendo instrumen. tos, cánulas, laringoscopios, conjuntos de ventilador, oxímetros, incubadoras, lavamanos, secadores y paredes. Sólo se obtuvieron cultivos positivos de un laringoscopio y un oxímetro que habian sido utilizados en un niffo con ACA y desinfectados, después de usar $y$ antes de tomar las muestras, con Clorospar al $0,5 \%$, de acuerdo a las normas de la unidad. Este hallazgo es similar a lo publicado en otros casos de in. fecciones intrahospitalarias ${ }^{3,10}$ y a raíz de él se injció la desinfección con glutaraldehido, resultando negativos todos los cultivos de control posteriores.

\section{Discusión}

El Acinetobacter calcoaceticus var. anitratus es una bacteria Gram negativa, de morfología variable; ha sido clasificada anteriormente en los géneros Bacterium, Herellea, Moraxella, Mi . ma, Achrobacter, Alcaligenes, Neisseria, Micrococcus, Diplococcus y Cytophaga. Actualmente, todas las cepas con diferencias menores $\mathrm{y}$ características bjoquímjcas o de crecimiento sinilares se incluyen reunidas en el género y especie Acinetobacter calcoaceticus ${ }^{\mathrm{It}}$. Su principal importancia radica en que es capaz de producir infecciones nosocomiales, especialmente en pa. cientes inmunocomprometidos ${ }^{12} y$ en unidades de cuidado intensivo de adultos, niños y recién nacidos ${ }^{1-3}$.

En nuestra unidad, ACA ha sido endémico desde mediados de la década de 1980 , pero en 1989 se transformó en uno de los principales agentes identificados en pacientes con infecciones intrahospitalarias, desplazando a otras bacterias de ocurrencia frecuente como Psetudomonas y Klebsiella, siendo sólo superado por Staphylococcus aureus multirresistente, y en el año siguiente causó el brote que se describe, con la inusitadamente elevada letalidad que señalamos, desconocida para este germen. No sabemos de otro brote semejante en el medio nacional, si bien en otros pajses se 10 menciona frecuentemente ${ }^{1-3,13}$, aunque no con la gravedad de nuestros casos. La mayor susceptibilidad de los varones a la infección ha sido descrita previamente ${ }^{14,15}$ Además, las mujeres tendrian menor frecuencia de enfermedad de la membrana hialina, Jo que contribuiría a reducir el riesgo de ingreso de sexo femenino a unidades de cuidados intensivos ${ }^{16} \mathrm{y}$, por lo tanto, la posjbilidad de complicaciones nosocomiales. La prematuridad, el peso bajo, la dificultad respiratoria, el antecedente de asfixia o Apgar bajo fueron los principales motivos por los cuales estos niños ingresaron a nuestra unidad. La característica de oportunista del ACA se revela en el hecho de haber afectado a recién nacidos sometidos a procedimientos que favorecen las infecciones intrahospitalarias ${ }^{13}, 17,18$, ventilacjón mecánica, la alimentación parenteral y antibioticoterapia previa.

Los síntomas y signos de septicemia y las manifestaciones hematológicas fueron similares a las de otras descripciones ${ }^{5,19,20}$.

$\mathrm{La}$ frecuencia de infecciones nosocomiales por ACA ha ido aumentando, como también su agresividad, como sugiere la mayor letalidad y al tiempo de latencia más breve de las septicemias ocurridas en 1990. El aumento de la resistencia de ACA a los antibióticos sólo puede ser explicado por la aparición de cepas resis. tentes, sin que podamos precisar el mecanismo de su adquisición, pero creemos que el uso indiscriminado de antibióticos en jas UCIN pueje ser un factor predisponente ${ }^{5}$. Es posible que la transmisión por otras vías que los instrumentos contaminados, como la colonización de personal, también juegue un rol importante en la diseminación del agente ${ }^{21,22}$, lo que no pudimos demostrar en nuestros pacientes. Desde mayo de 
Tabla

Caracter ísticas clínicas de los pacientes

con septicemia intrahospitalaria por ACA

\begin{tabular}{lccc}
\hline $\begin{array}{l}\text { Características } \\
\text { clinicas }\end{array}$ & año $1989(\mathrm{n}=10)$ & 1990 (n=11) & Ambos años (n=21) \\
\hline $\begin{array}{l}\text { Color terroso y, } 0, \\
\text { aspecto séptico }\end{array}$ & 8 & 10 & 18 \\
Hipotermia o fiebre & 8 & 8 & 16 \\
Maja tolerancia oral & 7 & 7 & 14 \\
Neumonía & 6 & 8 & 14 \\
Hepatomegalia & 7 & 7 & 14 \\
Lesiones cutáneas & 4 & 3 & 7 \\
Apneas y, o, bradicardia & 3 & 1 & 4 \\
Compromiso neurológico & 1 & 3 & 4 \\
Shock séptico & - & $4 *$ & 4 \\
\hline
\end{tabular}

* Corresponden a 4 casos con evolución fatal.

1990 hasta la fecha de esta publicación, no hemos vuelto a tener casos fatales de septicemia por ACA, pero creemos necesario tener presente, en las infecciones intrahospitalarias, la posibilidad que se trate de este germen, para tomar medidas que eviten su propagacjón y un brote epidémico de alto costo en vidas humanas.

\section{Resumen}

Se presentan 21 casos de septicemia por Acinetobacter calcoaceticus var anitratus, 10 ocurrieron en el año 1989 y 11 en 1990 (enero a abril), en este último año, 9 casos presentaron características de brote epidémico. Se destaca su predominio en hombres, de menos de $2500 \mathrm{~g}$ y menores de 37 semanas, que ingresaron a la UCDN por distintas causas, que en su mayoría requirieron ventilación mecánica, alimentación parenteral y recibieron antibióticos previamente. El hemograma y las caracteristicas clínicas no difieren de lo publicado para sepsis por atıos gérmenes. Se destaca el aumento no sólo en la frecuencia del ACA como agente nosocomial, sino que se advierte de su mayor agresividad y resistencia a antibióticos, lo que provocó una letajidad elevadísima ( 8 de 11 casos) en nuestra
UCIN. Finalmente, y gracias a que se tomaron severas medidas de control, se logró el cese del brote epidémico.

(Palabras clave: recién nacido, septicemia, Acinetobacter calcoaceticus.)

\section{Referencias}

1. Smego RA Jr: Endemic Nosocomial Acinetobacter Calcoaceticus Bacteremia. Clinical significance, treatment and prognosis. Arch Intern Med 1985: 145: $2174-2179$.

2. Sakata $H$, Fujita $K$, Maruyama $S$, Kakehashi $H$, Mori $Y$. Yoshioke $H$ : Acinetobacter calcoaceticus biovar anitratus septicaemia in a neonatal intensive care unit: epidemiology and control J Hosp Infect $1989 ; 14: 15-22$.

3. Srone $J$, Wand Dos BC: Investigation of an outbreak infection with $A C A$ in a special care baby unit. J Hosp Infect $1986 ; 7: 42-48$.

4. Rubin SJ, Granato PA, Woslilouskas BL: Bact Gram negativas que no fermentan la glucosi. En: Lennete EH, Manual de Microbiología Clínjca. $4^{\text {a }}$ Edición, Bs.As.: Edit. Panamericana 1987; $418-424$.

5. Klein JO, Marcy SW: Bacterial sepsis and meningitis. In: Remington JS, Klein JO. Infections Disease of Fetus and Newborn Infant. Philadelphia: WH Sainders 1983; 679-735.

6. García J: Henocultivo en: Procedimientos y técnicas de laboratorio: Microbiología General Vol. I, Santiago: Edit. Universitaria 1983, 31-39. 
7. Bauer $A \boldsymbol{w}$, Kirby $\boldsymbol{W N}$, Sherris $J C$, Tuck $\boldsymbol{H}$ : Artibiotic susceptibility testing by standardizing single dise method. Am J Clin Path 1966:45: 493-496.

8. Manroe BL, Weinberg $A G$, Rosenfeld $C R$, Browne $R$ : The neonatal blood count in health and disease I. Reference values for neutrophiljc cells. J Pediatr $1979 ; 95: 89.98$.

9. Ministerio de Salud: Nomas de Areas Críticas. Neonatologia Control de Infecciones Intrahospitalarias $1990 ; 1980$ (Cap. 3).

10. Zelada $\boldsymbol{M}$, Narorete $C$. Infección Vieonatal por Listeria Monocytogenes. Rev. Chil Pediatr 1990: $61: 17-24$.

11. Juni E: Acinetobacter. In: Bergey's. Manual of systematic Bacteriology. Baltimore: Williams and Wilkins, 1984; 303-307.

12. Nelson $J D$; Acinetobacter Infection. In: FeiginCherry Text Book of Pediatric Infections Diseases Second Edition Philadelphia: WB Saunders 198?; 1073-1074.

13. Larson $E$ : A decade of nosocomial Acjnetobacter. Am J Jnfect Control 1984; 12:14-18.

14. Washburn $T C$, Medearis $D N J$ and Childs $B$ : Sex differences in susceptibility to infections. Pediatrics 1965: 35: 57-64.
15. Schege! $R J$ and Bellanti $J A$ : Increased susceptibility of males to infection. Lancet $1969 ; 2$ : 826-829.

16. Naeye RL, Burt LS, Wright Dl.: Neonatal mortality the male disadvantage. Pediatrics 1971; 48: 902-906.

17. Goldman DA, Durbin WA Jr. Freeman J: Nosocomial infections in a neonatal intensive care unit. J Infect Dis $1981 ; 144: 449-459$.

18. Mayhall $C G$, Lamb $V A$, Bitar CM: Nosocomial KLbsiella infection in a neonatal unit: identification of risk factors for gastrointestinal colonization. Infec Control 1980: 1: 239-246.

19. Zelada $\boldsymbol{M}: \mathbf{E}$ l hemograma en las infecciones bacteriaras noenatales. Rey Chil Pediatr 1989; 60: 142-147.

20. Rodwell RI, Leslie AL, Tudehope DI: Homatologic scaring to diagnose neonatal sepsis. $J$ Pediat $1988 ; 112: 761-766$.

21. Hartstein A, Rashad A, Liebler JMet al.: Multiple ICU Acinetobacter Outbreak. American J Medicine 1988; 85: 624-631.

22. Guerero $O P$. Francote $N$. Pino $M$, Vaisman $S$ : Infección intrahospitalaria por Klebsiella Pneumonia en un brote de sepsis en recién nacidos. Rev Chil Pediatr 1981; 52 : 306-313. 